\title{
Extrapolating FR-0 Radio Galaxy Source Properties from Propagation of Multi-Messenger Ultra-High Energy Cosmic Rays
}

\author{
Jon Paul Lundquist, ${ }^{1, *}$ Lukas Merten, ${ }^{2}$ Serguei Vorobiov, ${ }^{1}$ Margot Boughelilba, ${ }^{2}$ \\ Anita Reimer, ${ }^{2}$ Paolo Da Vela, ${ }^{2}$ Fabrizio Tavecchio, ${ }^{3}$ Giacomo Bonnoli ${ }^{4}$ and Chiara \\ Righi $^{3}$ \\ ${ }^{1}$ Center for Astrophysics and Cosmology (CAC), University of Nova Gorica, Vipavska 13, SI-5000 Nova \\ Gorica, Slovenia \\ ${ }^{2}$ Institute for Astro and Particle Physics, University of Innsbruck, Technikerstraße 25, 6020 Innsbruck, \\ Austria \\ ${ }^{3}$ Astronomical Observatory of Brera, Via Brera 28, 20121 Milano, Italy \\ ${ }^{4}$ Instituto de Astrofísica de Andalucía (CSIC), Apartado 3004, E-18080 Granada, Spain \\ E-mail: jplundquist@gmail.com
}

\begin{abstract}
Recently, it has been shown that relatively low luminosity Fanaroff-Riley type 0 (FR-0) radio galaxies are a good candidate source class for a predominant fraction of cosmic rays (CR) accelerated to ultra-high energies (UHE, E $>10^{18} \mathrm{eV}$ ). FR-0s can potentially provide a significant fraction of the UHECR energy density as they are much more numerous in the local universe than more energetic radio galaxies such as FR-1s or FR-2s (up to a factor of $\sim 5$ with $\mathrm{z} \leq 0.05$ compared to FR-1s).

In the present work, UHECR mass composition and energy spectra at the FR-0 sources are estimated by fitting simulation results to the published Pierre Auger Observatory data. This fitting is done using a simulated isotropic sky distribution extrapolated from the measured FR-0 galaxy properties and propagating CRs in plausible extragalactic magnetic field configurations using the CRPropa3 framework. In addition, we present estimates of the fluxes of secondary photons and neutrinos created in UHECR interactions with cosmic photon backgrounds during CR propagation. With this approach, we aim to investigate the properties of the sources with the help of observational multi-messenger data.
\end{abstract}

$37^{\text {th }}$ International Cosmic Ray Conference (ICRC 2021)

July 12th - 23rd, 2021

Online - Berlin, Germany

\footnotetext{
*Presenter
} 


\section{Introduction}

Lower luminosity Fanaroff-Riley (FR-0) radio galaxies are a good candidate source class for a predominant fraction of cosmic rays (CR) accelerated to ultra-high energies (UHE, E $>10^{18} \mathrm{eV}$ ). Using the average FR-0 spectral energy distribution (SED), it was found [1] they can accelerate UHECR up to the highest observed energies via hybrid acceleration (e.g., combining Fermi-I preacceleration with gradual shear acceleration). FR-0s may thus provide a significant fraction of the UHECR energy density as they are much more numerous in the local universe than more energetic FR-1 or FR-2 radio galaxies (by a factor of $\sim 5$ compared to FR-1s) [2].

Further investigation of FR-0 source properties can be done using multi-messenger observations. In this work, isotropic FR-0 UHECR emission was simulated, extrapolated from measured FR-0 properties, and propagated to Earth in plausible extragalactic magnetic fields using CRPropa3 [3]. Fitting this simulation to Pierre Auger Observatory data [4, 5] estimates UHECR mass composition and energy spectra emitted at FR-0s. Additionally, we present estimates of secondary photon and neutrino fluxes created by UHECR interactions with cosmic photon backgrounds.

\section{Isotropic FR-0 Simulation}

The simulated FR-0 set is upsampled from the FR0CAT catalog [2] well-sampled sky portion in the supergalactic coordinate spherical rectangle of $-45^{\circ}$ to $45^{\circ} \mathrm{SGB}$ and $60^{\circ}$ to $120^{\circ} \mathrm{SGL}$, as shown in Figure 1 on the left. There are 76 out of 104 FR-0s in this $11.79 \%$ sky section; this results in 645 upsampled FR-0s distributed isotropically in the simulation (Figure 1 on the right).

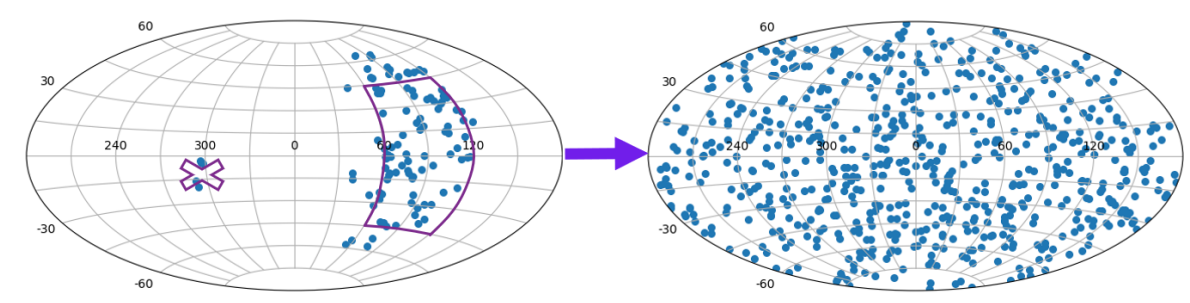

Figure 1: The FR-0 catalog FR0CAT [2] in supergalactic coordinates (left) and the simulated FR-0 distribution (right). Full-sky isotropic FR-0 density is estimated from the well-sampled FR0CAT section.

Isotropic simulated FR-0 have the same redshift distribution as data (Figure 2a) and the relative flux distribution is proportional to the radio output distribution (Figure 2b). Furthermore, the simulated set preserves the correlation between radio (a source jet power measure)/UHECR flux and redshift (Figure 2c) to model the local universe source evolution. The flux of each simulated source is emitted isotropically (approximating that FR-0 possess relatively slow bulk flows).

\section{Propagating UHECRs through the Extragalactic Environment}

The CRPropa3 (v3.1.6) framework [3] is used to simulate the propagation of proton, nitrogen, and iron UHECR primaries through the extragalactic environment. These nuclei are thrown with an energy spectrum $d N / d E \propto E^{-1}$ with energies from $10^{18.6}$ to $10^{21} \mathrm{eV}$. 


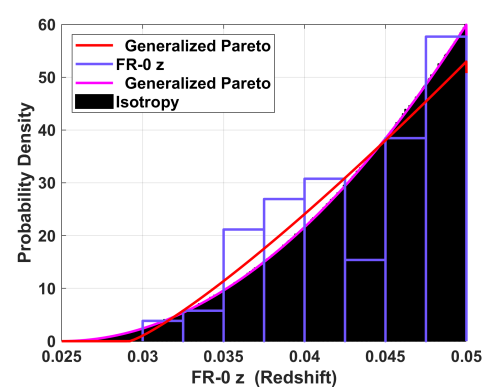

(a)

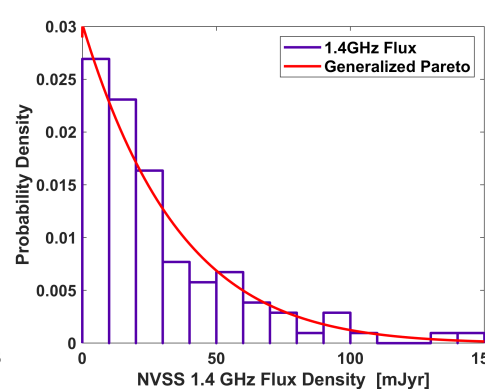

(b)

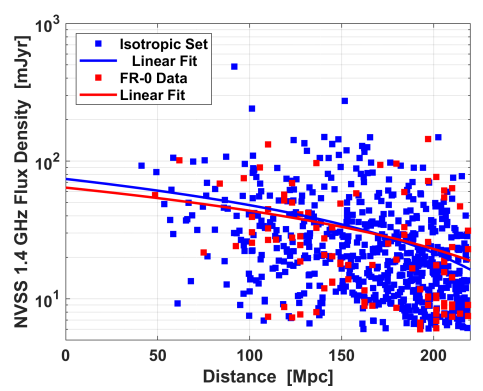

(c)

Figure 2: (a) Simulated FR-0 redshift generated by Pareto distribution fit to catalog data [2]. Data redshift isotropy probability is $\sim 16 \%$. (b) Relative simulated FR-0 UHECR flux is proportional to radio output distribution. Generated by Pareto distribution fit to NVSS data [2]. (c) Local universe source evolution modeled by preserving redshift/flux correlation (Kendall's correlation coefficient: -0.28 , p-Value: $4.6 \times 10^{-5}$ ) [2].

\subsection{UHECR Interactions}

Simulated UHECR interactions with the extragalactic radiation backgrounds CMB, IRB (Gilmore12 model [6]), and URB (Protheroe96 model [7]) include: Photo-pion production and nuclear photodisintegration (GZK effect), Pair-production (including double and triplet), and Inverse Compton scattering. Furthermore, adiabatic cooling due to redshift and nuclear decays of unstable particles are also included.

\subsection{Magnetic Fields}

Magnetic field deflections are simulated in CRPropa3 using a turbulent magnetic field (via the initTurbulence module) with a mean field strength of $1 \mathrm{nG}$ and length scales from $60 \mathrm{kpc}$ to $1 \mathrm{Mpc}$ (or $3 \mathrm{Mpc}$ ) on an isotropic Kolmogorov power spectrum with an average $234 \mathrm{kpc}$ (or $647 \mathrm{kpc}$ ) correlation length. The magnetic field strength distribution is shown in Figure 3. Particles are propagated through this field using the PropagationCK module with a propagation step size minimum of $10 \mathrm{kpc}$ to a maximum of $4 \mathrm{Mpc}$ with a 0.01 iterative calculation error tolerance.

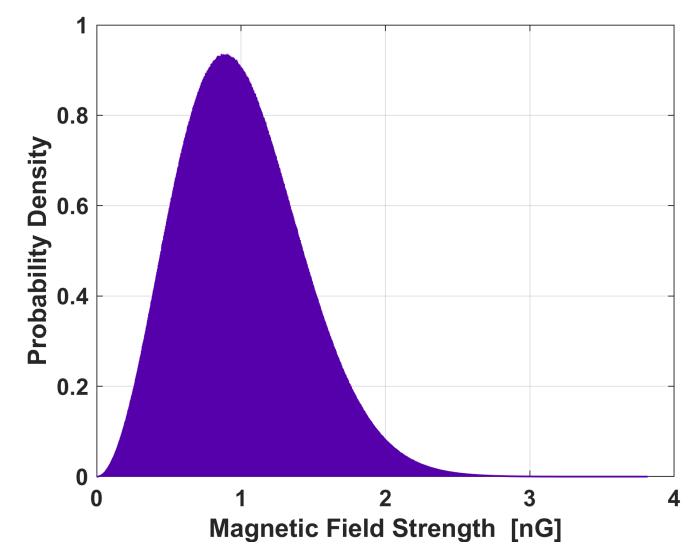

Figure 3: Distribution of simulated magnetic field strengths for an average $\langle B\rangle=1 \mathrm{nG}$ and $\sqrt{\left\langle B^{2}\right\rangle}=1.1 \mathrm{nG}$. 


\section{Isotropic FR-0 Simulation Output}

The particle observer is a $200 \mathrm{kpc}$ sphere at the simulated extragalactic magnetic field center. Nuclei intersecting this sphere are recorded and are not propagated further. Nuclei below the energy threshold of $10^{18.6} \mathrm{eV}$ at any point during propagation are discarded. Particles that have travelled further than $2000 \mathrm{Mpc}$, or intersect a sphere $2000 \mathrm{Mpc}$ around the observer, are also discarded.

Secondary photons above an energy of $100 \mathrm{MeV}$ are immediately recorded, if their path intersects the observer sphere, for post-processing propagation using the DintElecaPropagation module [8,9]. The crossover energy from EleCa to DINT is $100 \mathrm{TeV}$. Secondary neutrinos above an energy threshold of $100 \mathrm{TeV}$ are immediately recorded if their path intersects the observer sphere.

\subsection{Composition Output}

Figures of mean $\log$ mass number $\langle\ln A\rangle$ versus detected energy are shown here for thrown nitrogen (Figure 4) and iron (Figure 5) nuclei. After propagation, the detected mass distributions of the thrown nitrogen and iron are modified due to spallation against the photon backgrounds and nuclear decay (proton is of course unchanged). A shift to smaller mass numbers can be seen for larger maximum coherence scale fields due to the longer average propagation time.
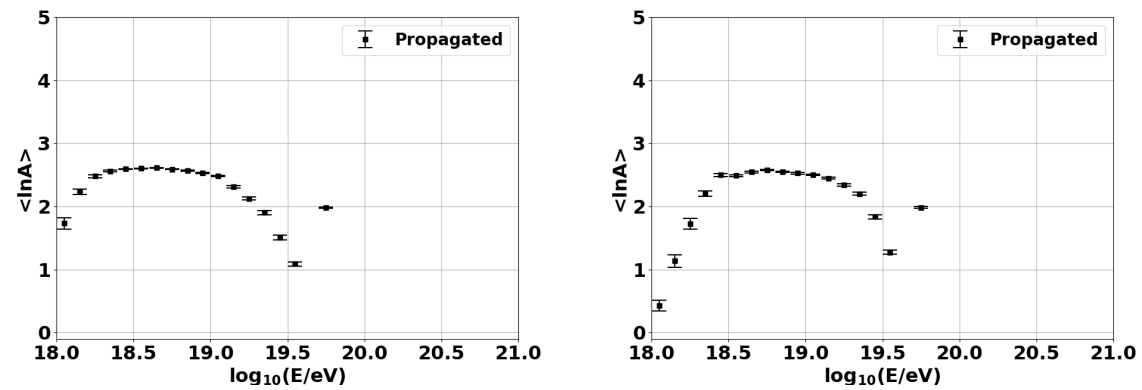

Figure 4: Nitrogen $(\ln (\mathrm{A})=2.6)$ detected $\langle\ln A\rangle$ vs. energy. (a) $1 \mathrm{Mpc}$ maximum scale magnetic field (b) $3 \mathrm{Mpc}$ maximum scale magnetic field. Larger coherence scale fields result in smaller mass numbers.
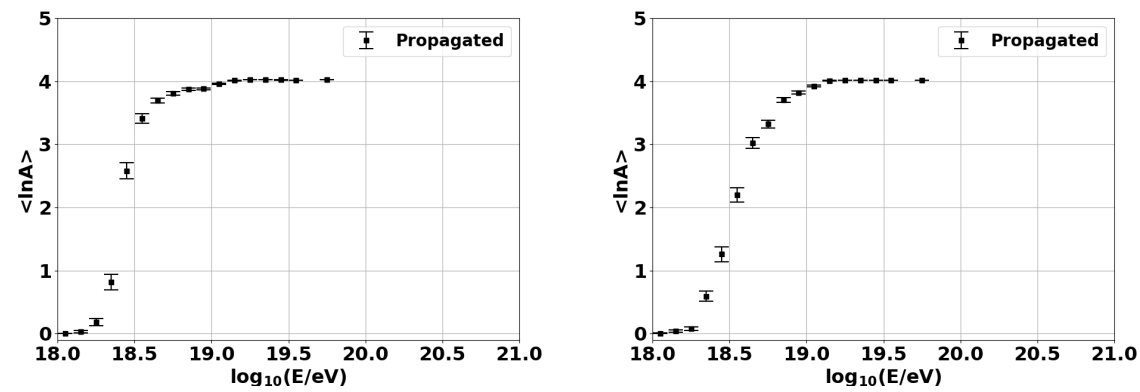

Figure 5: Iron $(\ln (\mathrm{A})=4.0)$ detected $\langle\ln A\rangle$ vs. energy. (a) $1 \mathrm{Mpc}$ maximum scale magnetic field. (b) $3 \mathrm{Mpc}$ maximum scale magnetic field. Larger coherence scale fields result in smaller mass numbers.

\subsection{Energy Spectra Output}

The detected relative energy spectra for the thrown three primary nuclei, for each scale magnetic field, after propagation are shown in Figure 6 (proton), Figure 7 (nitrogen), and Figure 8 (iron). The thrown energy spectrum $d N / d E \propto E^{\gamma}$ spectral index is $\gamma=-1$. After propagation, the energy 

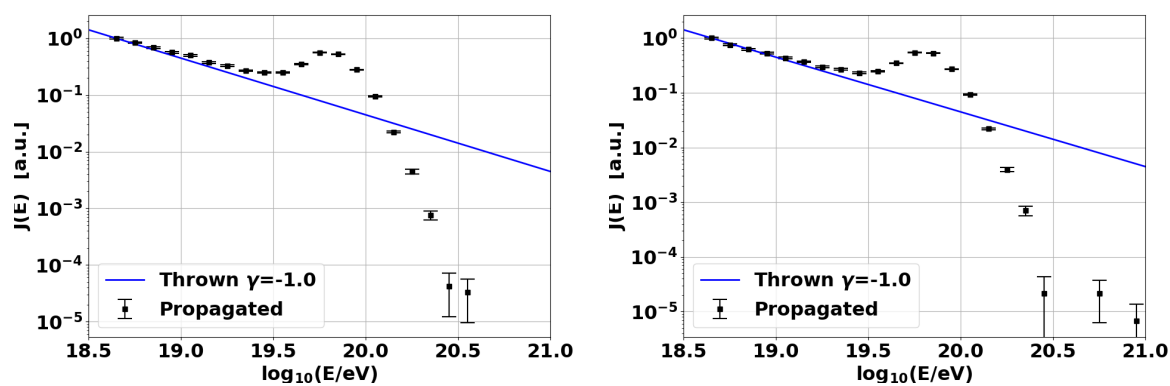

Figure 6: Relative energy spectra of proton thrown with $d N / d E \propto E^{-1}$ after propagation. (a) $1 \mathrm{Mpc}$ maximum scale magnetic field. (b) $3 \mathrm{Mpc}$ maximum scale magnetic field.
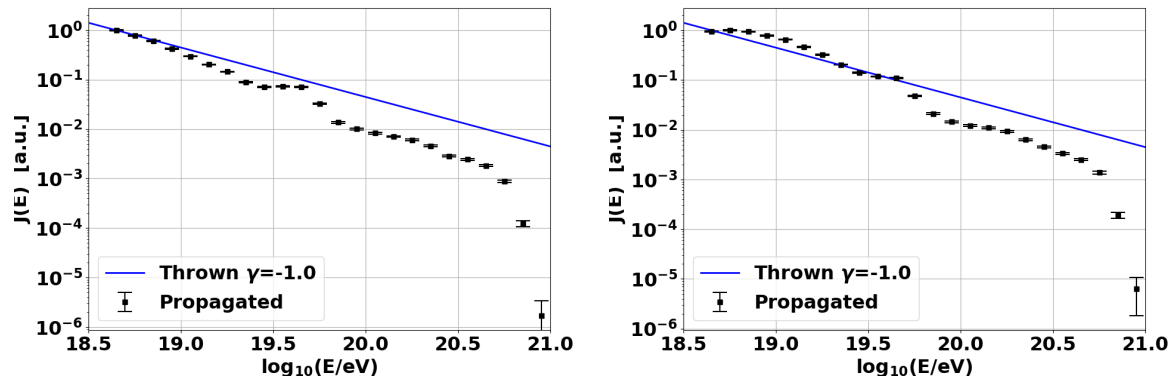

Figure 7: Relative energy spectra of nitrogen thrown with $d N / d E \propto E^{-1}$ after propagation. (a) $1 \mathrm{Mpc}$ maximum scale magnetic field. (b) $3 \mathrm{Mpc}$ maximum scale magnetic field. A small excess at lower energies can be seen for the larger coherence scale field.
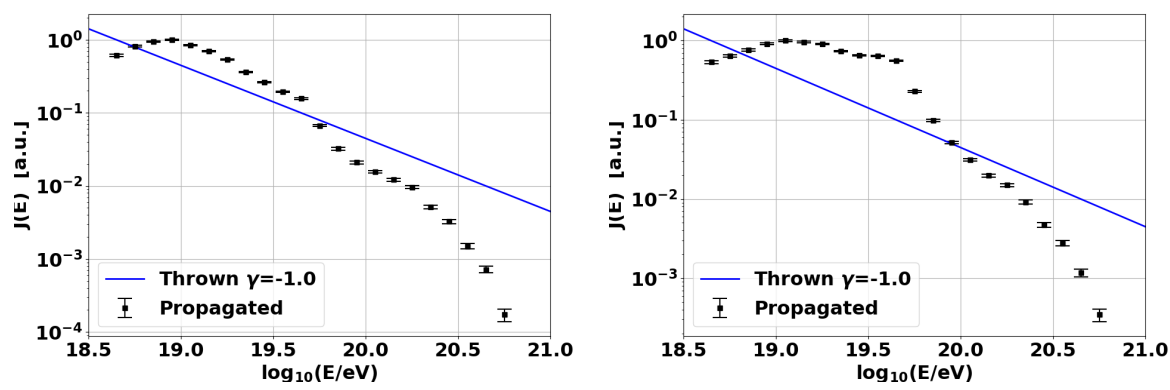

Figure 8: Relative energy spectra of iron thrown with $d N / d E \propto E^{-1}$ after propagation. (a) 1 Mpc maximum scale magnetic field. (b) $3 \mathrm{Mpc}$ maximum scale magnetic field. A more pronounced excess at lower energies can be seen for the larger coherence scale field.

spectra are modified by interactions with the extragalactic environment. A larger excess of lower energy nuclei can be seen for the larger coherence scale magnetic field ( $3 \mathrm{Mpc}$ max.) and this excess increases with increasing initial primary UHECR mass. This shift to lower energies is due to the longer average propagation time for larger coherence scale fields.

\section{FR-0 Source Emission Result}

\subsection{FR-0 Source Composition Result}

The propagation modified $\langle\ln A\rangle$ and energies are used to calculate the relative proportions of FR-0 source emitted nuclei by the best fit to Pierre Auger Observatory data [4]. These percentages 
are then used to calculate the average percentage of each thrown nuclei in bins of emitted energies, as shown in Figure 9 and Figure 10 for the two fields considered. The error bars are bootstrapped $1 \sigma$ uncertainties taking into account data uncertainties and simulation statistics.
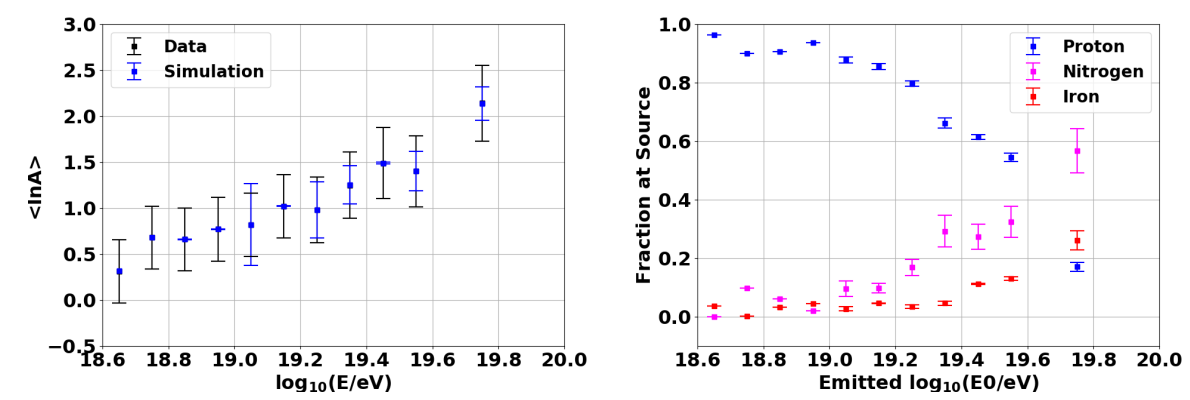

Figure 9: 1 Mpc max. scale field result. (a) Pierre Auger Observatory ICRC 2019 QGSJETII-04 data fit [4]. (b) Simulated FR-0 sources emitted primary nuclei percentages versus energy. The last bin is $E \geq 10^{19.6}$.
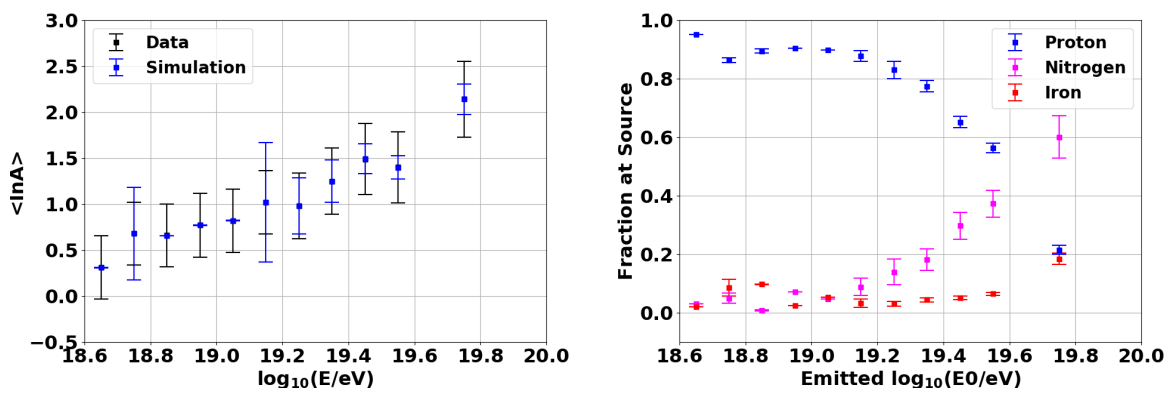

Figure 10: 3 Mpc max. scale field result. (a) Pierre Auger Observatory ICRC 2019 QGSJETII-04 data fit [4]. (b) Simulated FR-0 sources emitted primary nuclei percentages versus energy. The last bin is $E \geq 10^{19.6}$.

\subsection{FR-0 Source Energy Spectrum Result}

Using the resulting composition mix, $\chi^{2}$ is minimized for the best fit to Pierre Auger Observatory's ICRC 2019 energy spectrum $\left(\mathrm{E}>10^{18.6} \mathrm{eV}\right)$ [5] by re-weighting thrown energies and flux normalization. The result is an estimated FR-0 source emission energy spectral index, see Figure 11. The relatively soft emitted spectra result may be connected to the lack of a rigidity dependent cut-off in the fitting and the inclusion of an IGMF in our simulations, see $[10,11]$ for comparison.

Repeating the fitting on a set of simulations with no magnetic field present results in a poor fit to the energy spectrum due to an excess above data for energies $\mathrm{E}>10^{19.7} \mathrm{eV}$.

\subsection{Multi-messenger Spectra}

Preliminary secondary multi-messenger particle energy spectra are shown in Figure 12. The $1 \mathrm{Mpc}$ maximum scale magnetic field mixed composition and source spectral index fit results are used. Photon output shown in Figure 12a is from the DintElecaPropagation module [8,9].

The resulting cosmogenic all-neutrino spectrum shown in Figure $12 \mathrm{~b}$ appears similar to other results with soft spectra sources such as in [12] which used only protons. 

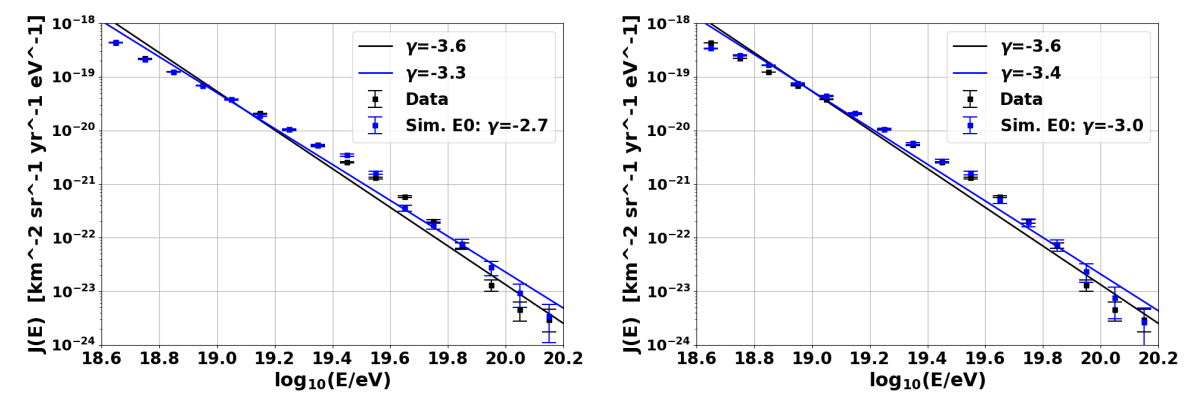

Figure 11: Mixed composition best fit to the Pierre Auger energy spectrum [5]. (a) $1 \mathrm{Mpc}$ max. scale field. (b) 3 Mpc max. scale field.

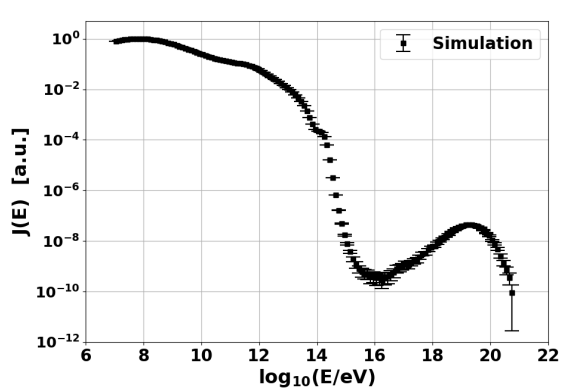

(a)

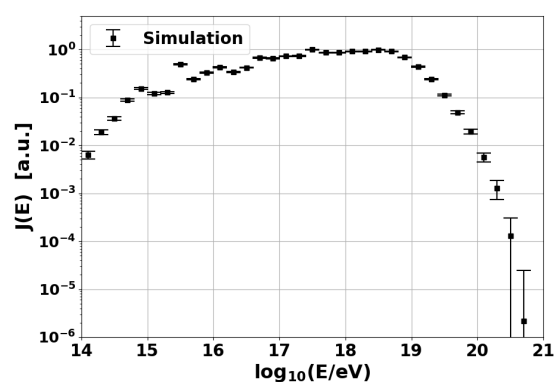

(b)

Figure 12: Simulated secondary multi-messenger relative energy spectra: (a) Photons. (b) Neutrinos.

\section{Conclusion}

Fanaroff-Riley (FR-0) radio galaxies have been shown to be capable of accelerating cosmic rays to ultra-high energies $\left(\mathrm{E}>10^{18.6} \mathrm{eV}\right)[1,2]$. Fitting isotropic FR-0 simulations extrapolated from measured FR-0 properties in reasonable extragalactic fields to published Pierre Auger Observatory UHECR data $[4,5]$ has been done. The presented comparison of simulated FR-0 source mass composition and cosmic ray energy spectra to UHECR data results in good fits. This insight into possible FR-0 emission properties results in a soft source energy spectrum spectral index of $\gamma \sim-2.7$ along with an increase in the emitted nuclei masses with increasing energy. Additionally, the estimated shapes of the secondary photon and neutrino fluxes created by UHECR interactions with cosmic photon backgrounds are reasonable. Future work will include the addition of a rigidity dependent exponential cutoff, additional magnetic field configurations, and estimates of the required FR-0 CR luminosity.

\section{Acknowledgements}

Financial support was received from the Austrian Science Fund (FWF) under grant agreement number I 4144-N27 and the Slovenian Research Agency - ARRS (project no. N1-0111). MB has for this project received funding from the European Union's Horizon 2020 research and innovation program under the Marie Sklodowska-Curie grant agreement No 847476. The views and opinions expressed herein do not necessarily reflect those of the European Commission. G.B. acknowledges 
financial support from the State Agency for Research of the Spanish MCIU through the "Center of Excellence Severo Ochoa" award to the Instituto de Astrofísica de Andalucía (SEV-2017-0709) and from the Spanish "Ministerio de Ciencia e Innovación" (MICINN) through grant PID2019107847RB-C44. The Slovenian National Supercomputing Network provided extensive computing support.

\section{References}

[1] Merten, L., Boughelilba, M., Reimer, A., Da Vela, P., Vorobiov, S., Tavecchio, F., Bonnoli, G., Lundquist, J.P., Righi, C., Astropart. Phys. 128 (2021) 102564. arXiv:2102.01087, doi: $10.1016 /$ j . astropartphys.2021. 102564.

[2] Baldi, R. D., Capetti, A., Massaro, F., Astron. Astrophys. 609 (2018) A1. arXiv : 1709. 00015 , doi: $10.1051 / 0004-6361 / 201731333$.

[3] Alves Batista, R., Dundovic, A., Erdmann, M., Kampert, K.H., Kuempel, D., Müller, G., Sigl, G., van Vliet, A., Walz, D., Winchen, T., JCAP 05 (2016) 038. arXiv: 1603.07142, doi : $10.1088 / 1475-7516 / 2016 / 05 / 038$.

[4] Yushkov, A. on behalf of the Pierre Auger Collaboration, PoS ICRC2019 (2020) 482. doi: $10.22323 / 1.358 .0482$.

[5] Deligny, O., PoS ICRC2019 (2020) 234. arXiv: 2001.08811, doi : 10.22323/1.358. 0234.

[6] Gilmore, R.C. and Somerville, R.S. and Primack, J.R. and Domínguez, A., Mon. Not. R. Astron. Soc. 422 (4) (2012) 3189-3207. arXiv: 1104.0671v2, doi : 10.1111/j .1365-2966. 2012.20841.x.

[7] Protheroe, R.J. and Biermann, P.L., Astropart. Phys. 6 (1996) 45-54, [Erratum: Astropart.Phys. 7, 181 (1997)]. arXiv: astro-ph/9605119, doi : 10 . 1016/S0927-6505 (96) 00041-2.

[8] Settimo, M. and De Domenico, M., Astropart. Phys. 62 (2015) 92-99. arXiv: 1311.6140, doi: $10.1016 / \mathrm{j}$. astropartphys .2014 .07 .011$.

[9] Lee, S., Phys. Rev. D 58 (1998) 043004. arXiv:astro-ph/9604098, doi:10.1103/ PhysRevD. 58.043004.

[10] Aab, A., et al., JCAP 04 (2017) 038, [Erratum: JCAP 03, E02 (2018)]. arXiv: 1612 .07155, doi : $10.1088 / 1475-7516 / 2017 / 04 / 038$.

[11] Heinze, J., Fedynitch, A., Boncioli, D., Winter, W., Astrophys. J. 873 (1) (2019) 88. arXiv: 1901.03338, doi: 10.3847/1538-4357/ab05ce.

[12] van Vliet, A. and Alves Batista, R. and Hörandel, J.R., Phys. Rev. D 100 (2) (2019) 021302. arXiv: 1901.01899, doi: 10.1103/PhysRevD. 100.021302. 